\title{
A Strong Transcription Activity of the Bombyx mori Elongation Factor $1 \alpha$ Promoter
}

\author{
Tae-Won Goo, Sung-Wan Kim, Seong-Ryul Kim, Seung-Won Park, Seok-Woo Kang, Kwang-Ho Choi, and \\ Eun-Young Yun* \\ Department of Agricultural Biology, National Academy of Agricultural Science, RDA, Suwon, 441-100, Republic of Korea
}

(Received 15 May 2012; Accepted 28 June 2012)

\begin{abstract}
We previously isolated 9 clones that show stronger signal compared to $B$. mori cytoplasmic actin gene (BmA3) by using a dot blot hybridization. In this study, we focused on one clone among these clones which has high amino acid homology with elongation factor $\alpha$ gene of $B$. mori. This clone, named bEF1 $\alpha$ ( $B$. mori elongation factor $\alpha$ ) was ubiquitously expressed in all tissues and developmental stage of $B$. mori. As result of promoter assay using dual luciferase assay system, we found the highest transcription activity region $(-702 /+38)$ in the 5 '-flanking region of bEF1 $\alpha$ gene, which has about 20 fold more intensive promoter activity than BmA3 promoter. Moreover, the bEF1 $\alpha$ promoter was normally regulated in $\mathrm{Bm5}, \mathrm{Sf} 9$, and $\mathrm{S2}$ cells. Therefore, we suggest that bEF1 $\alpha$ promoter may be used more powerful and effectively for transgene expression in various insects containing $B$. mori as a universal promoter.
\end{abstract}

Key words: Bombyx mori, Elongation factor $1 \alpha$, Luciferase assay, Promoter

\section{Introduction}

Advance in biotechnology has made it possible to express heterologous genes in organisms. Numerous therapeutic proteins of human have been synthesized by expressing their genes in various host systems. The domestic silkworm, $B$. mori as a bioreactor is one of the most attractive

\footnotetext{
*To whom the correspondence addressed

Dr. Eun-Young Yun, Department of Agricultural Biology, National Academy of Agricultural Science, RDA, Suwon 441100 , South Korea.

Tel: +82-31-290-8576; Fax: +82-31-290-8543;

E-mail: yuney@korea.kr
}

host system for the production of recombinant proteins (Wurm, 2003). B. mori is uniquely suited for recombinant protein expression because of the following reasons: transgenesis was recently achieved using transposon piggyBac and minos (Tamura et al., 2000; Uchino et al., 2007); aseptic mass rearing system is available all the year round; its cells have mimic the complex post-translational modification processes of mammalian cells, such as glycosylation, disulfide bond formation, and protolytic processing (Ailor and Betenbaugh, 1999; Jacobs and Callewaert, 2009; Kato et al., 2010).

Silkworm transgenesis is now a routine method leading to satisfactory yield of transformed animals and to a reliable expression of transgenes during multiple successive generations (Tamura et al., 2000; Uchino et al., 2007; Zhao et al., 2009). For the expression of transgenes, piggyBac vectors have already been incorporated with seven promoters: the transposon promoter itself (Handler et al., 1998), the artificial 3XP3 (Thomas et al., 2002), the Drosophila melanogaster hsp70 (Uhlírová et al., 2002), the B. mori cytoplasmic actin A3 (Tamura et al., 2000), and $B$. mori silk gland-specific promoters such as the heavy and light chain of fibroin (Tomita et al., 2003), the sericin 1 (Tomita et al., 2007), and the fibrohexamerin/ P25 (Royer et al., 2005). Among five promoters originated from $B$. mori, only BmA3 promoter can express marker genes and heterologous genes in most developmental stages and tissues (Mangé et al., 1997). In most case, for stable germline transformation of B. mori, BmA3 promoter was used to choose to drive the enhanced green fluorescent protein (EGFP) in piggyBac vector. The BmA3-EGFP marker was useful for the screening of G1 transgenic individuals at the larval stage, but not detectable in G0 embryonic tissues or in the eggs of the later generations (Thomas et al., 2002). Therefore, it is impossible to select transgenic individuals before the G1 larval stage using $\mathrm{BmA} 3$ promoter. Furthermore, the $\mathrm{BmA} 3$ pro- 
Table 1. Primers used to promoter constructs. The $M l u$ I sites in forward primers and Xho I sites in the reverse primers are underlined. The position of the primers is related to the transcription start site in bEF1 $\alpha$ gene

\begin{tabular}{|c|c|c|c|c|}
\hline Name & Position & Sequences & $\begin{array}{l}\text { Forward/ } \\
\text { reverse }\end{array}$ & Construct name \\
\hline D1 & $-905 /-885$ & 5'-CTCGAGCTGTCAACT CTCAAAATTT G-3' & $\mathrm{F}$ & pGL3-bEF1 $\alpha-D 1$ \\
\hline D2 & $-702 /-682$ & 5'-CTCGAGAGAATTTATTTTCCTAATTTG-3' & $\mathrm{F}$ & pGL3-bEF1 $\alpha-\mathrm{D} 2$ \\
\hline D3 & $-504 /-484$ & 5'-CTCGAGTGGGCTAATCAGCTAATGCTA-3' & $\mathrm{F}$ & pGL3-bEF1 $\alpha$-D3 \\
\hline D4 & $-244 /-222$ & 5'-CTCGAGGATTTTTAGTAAGATTTGTA-3' & $\mathrm{F}$ & pGL3-bEF1 $\alpha-\mathrm{D} 4$ \\
\hline D5 & $-98 /-78$ & 5'-CTCGAGCAGAAACAATTTGAAGGTTTT-3' & $\mathrm{F}$ & pGL3-bEF1 $\alpha-\mathrm{D} 5$ \\
\hline R1 & $+1 /+38$ & 5'-AGATCTCACGGATTACCAACTGCGTGT-3' & $\mathrm{R}$ & \\
\hline
\end{tabular}

moter is predominantly active in the midgut, which makes it difficult to reliably identify transformants since autofluorescence of many insect foods can mask low-level fluorescence (Horn et al., 2002)

Recently, we isolated 9 clones that show stronger signal compared to BmA3 by using a dot blot hybridization, and demonstrated ubiquitous and powerful promoter activity for two clones which encodes a heat shock protein 70 (bHsp70) and hypothetical protein 32 (bHp32), respectively (Goo et al., 2010; Goo et al., 2011).

In order to develop more strong promoters as compared to $\mathrm{BmA} 3$ promoter, we selected one clone among previously isolated 9 positive clones, which has high amino acid homology with elongation factor $1 \alpha$ gene of $B$. mori. In addition, its promoter was successfully isolated, and its potential and characteristics as constitutive promoter were investigated and compared to $\mathrm{BmA} 3$ promoter in this study.

\section{Materials and Methods}

\section{Experimental insects and cell lines}

The silkworm, B. mori (Jam 124) were reared on an artificial diet at $25^{\circ} \mathrm{C}, 65 \pm 5 \%$ relative humidity, and $12 \mathrm{~h}$ light : $12 \mathrm{~h}$ dark photoperiod. Bm5 cell lines derived from B. mori and Sf9 cell lines derived from Spodoptera frugiferda were maintained as adherent cell culture in TC100 insect medium (Sigma, USA) with 10\% (v/v) heatinactivated fetal bovine serum (Invitrogen, USA), as described previously [13]. Schneider S2 cell lines derived from $D$. melanogaster were maintained as adherent cell culture in Drosophila-SFM medium with $90 \mathrm{ml} / 1$ of $200 \mathrm{mM}$ L-glutamine and $5 \mathrm{ml} / 1$ of penicillin-streptomycin (Invitrogen, USA). Bm5 cells were subcultured with interval of 5-7 days and Sf9 and S2 cells subcultured with interval of 2-3 days.

\section{Screening of bEF1 $\alpha$ genomic DNA}

We screened genomic library constructed from $B$. mori larvae using the $\lambda$ DASH Vector Kit (Stratagene, USA). To prepare a probe, a $B$. mori heat shock protein 70 (bEF1 $\alpha$ ) cDNA was labeled with $\left[\alpha-{ }^{32} \mathrm{P}\right]$ dATP using a Random Primer Labeling Kit (Stratagene, USA). We obtained a positive clone in the 3rd screening. Phage DNA was extracted and digested with EcoR I, Xba I and $E c o$ R I/Xba I enzymes. The digested DNA fragments were subcloned into pUC18 vector. The nucleotide sequences of subcloned DNA fragments were determined using ABI 377 automatic sequencer (Perkin-Elmer, USA).

Relative Quantification analysis of mRNA by RT-PCR Total RNAs were prepared using RNA STAT-60 (TelTest, USA) according to the manufacturer's instruments and reverse transcribed with oligo (dT) primer using cDNA using High Capacity cDNA Archive Kit (Applied Biosystems, USA). After cDNA synthesis, RT-PCR was performed with a PCR Thermal Cycler Dice (TaKaRa, Japan) for $4 \mathrm{~min}$ at $95^{\circ} \mathrm{C}$, followed by 25 cycles of $20 \mathrm{~s}$ at $95^{\circ} \mathrm{C}, 30 \mathrm{~s}$ at $55^{\circ} \mathrm{C}$, and $25 \mathrm{~s}$ at $72^{\circ} \mathrm{C}$, followed by a final extension at $72^{\circ} \mathrm{C}$ for $10 \mathrm{~min}$.

\section{Prediction for transcription start site}

To identify the transcription start site of $b$ bEF $1 \alpha$ mRNA, 5'-RACE PCR was carried out with poly $\left(\mathrm{A}^{+}\right) \mathrm{RNA}$ using CapFishing ${ }^{\mathrm{TM}}$ Kit (Seegene, Korea) according to manufacturer's instructions. The adapter sequence attached to the ends of the cDNA enabled it to be used in 5'- RACE. Three gene-specific primers were designed using the sequences of the $b$ bEF $1 \alpha$ fragment. These primers were used in 5'RACE in conjunction with the anchor primer (5'-RACE primer: 5'-AGTGAAGGCCACGTTATGAAGGTGTGATGCG-3') to amplify the 5'-ends of cDNA. The PCR conditions were as followed: initial denaturation at $94^{\circ} \mathrm{C}$ for $3 \mathrm{~min}$ followed by 35 cycles of denaturation at $94^{\circ} \mathrm{C}$ for $40 \mathrm{~s}$, annealing at $68^{\circ} \mathrm{C}$ for $40 \mathrm{~s}$ and elongation at $72^{\circ} \mathrm{C}$, and a final elongation step at $72^{\circ} \mathrm{C}$ for $5 \mathrm{~min}$.

Plasmid construction and luciferase assay

The Dual Luciferase Report Assay System (Promega, 
USA) was used to measure promoter activity. For this assay, 5 serial deleted fragments of the 5'-flanking region of $b$ bEF $1 \alpha$ were generated by PCR amplification with primers listed in Table 1, and the PCR products were inserted into pGL3-Basic vector digested with Mlu I and Xho I sites (Promega, USA). The resulting experimental plasmids, pGL3-bEF1 $\alpha$-D1 (-905/+38), pGL3-bEF1 $\alpha$-D2 (-702/+38), pGL3-bEF1 $\alpha$-D3 (-504/+38), pGL3-bEF1 $\alpha-$ D4 (-244/+38), and pGL3-bEF1 $\alpha$-D5 (-98/+38) were generated. For normalization of transfection efficiency, the pRL-dHsp70-Rluc plasmid expressing Renilla luciferase under the regulation of the D. melanogaster Hsp70 (dHsp70) promoter was constructed.

Approximately $5 \times 10^{5}$ cells per well were plated in 24 well plates 1 day before transfection. Confluence of $50-$ $80 \%$ was achieved at 1 day after incubation, which is suitable to obtain high transfection efficiency. For transfection, $400 \mathrm{ng}$ of the experimental plasmid and $40 \mathrm{ng}$ of the pRL-dHsp70-Rluc control plasmid per well were mixed with $2 \mu$ of FuGENE HD Transfection Reagent (Roche, Germany) in TC-100 insect medium (Sigma, USA) for $15 \mathrm{~min}$ at room temperature. The mixture of DNA plasmids and FuGENE reagent was then applied to the appropriate well in triplicate. The transfected cells were incubated at $27^{\circ} \mathrm{C}$ for $48 \mathrm{~h}$, and harvested in $100 \mu \mathrm{l}$ of $1 \times$ passive lysis buffer (Promega, USA) per well. The transcriptional activities of firefly and Renilla luciferase were determined on a luminometer (TECAN, Switzerland) using Dual Luciferase Report Assay System (Promega, USA) according to the manufacturer's instruments. The luciferase activity of each experimental plasmid was normalized to Renilla activity.

\section{Results and Discussion}

\section{Selection of highly expressed cDNA clones compared to $\mathbf{B m A 3}$}

We previously isolated 9 clones that show stronger signal compared to B. mori cytoplasmoc actin gene (BmA3) by using a dot blot hybridization, and partially sequenced 9 positive cDNA clones and produced expressed sequence tags (ESTs) (Goo et al., 2011). In this study, we focused on the clone, which has high homology (99\%) with elongation factor $1 \alpha$ of $B$. mori.

\section{Structure analysis of full-length bEF1 $\alpha$ cDNA}

The complete nucleotide sequence (Genbank accession number: JQ638952) of the identified clone had a 5'untranslational region of 49 nucleotides followed by an initiating ATG codon. The TAA termination codon is at nucleotide 2,027, a consensus polyadenylation signal is
A

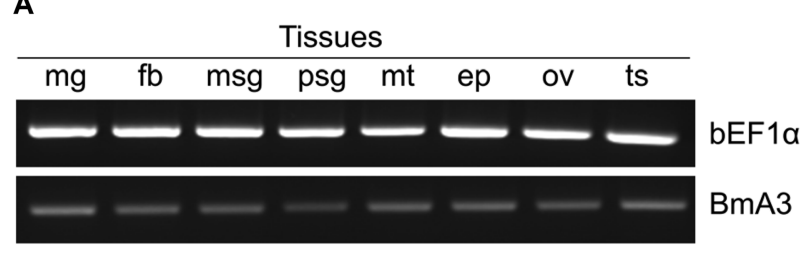

B

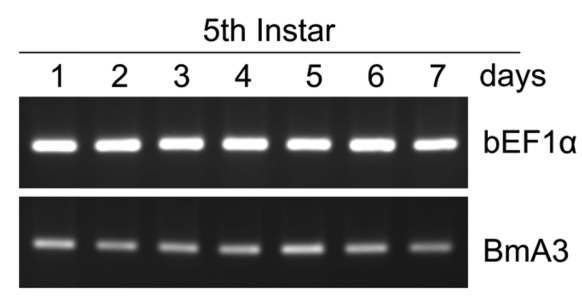

Fig. 1. Expression pattern according to tissue and development stage of bEF $1 \alpha$ mRNA. For analysis of mRNA expression on tissue (panel A), 1st-strand cDNA was synthesized from organs as follows: mg, midgut; fb, fatbody; msg, middle silk gland; psg, posterior silk gland; mt, Malpighian tubles; ep, epidermis; ov, ovary; ts, testis. For analysis of mRNA expression on developmental stage (panel B), 1st-strand cDNA was synthesized from day $1,2,3,4,5,6$, and 7 of 5 th instar B. mori larvae. RT-PCR was carried out using 1st-strand cDNAs synthesized from panel A and B.

present at 2,140-2,145, and the poly (A) tail is 18 nucleotides downstream of polyadenylation signal sequence (data not shown). Translation of the sequence from nucleotide 50 to 2,026 produces a 463-residue protein with a calculated $\mathrm{Mw}$ of $50.4 \mathrm{kDa}$ (Fig. 2). We named this clone as bEF1 $\alpha$ (B. mori elongation factor $1 \alpha$ ) because it has high homology (99\%) with elongation factor $1 \alpha$ of $B$. mori. EF-1 $\alpha$ promoters the GTP dependent binding of aminoacyl-tRNA to A site of ribosome (Hershey, 1991). The amino acid sequence of EF- $1 \alpha$ proteins is highly conserved between different species, a conservation which is particularly striking in N-terminal half, where the GTP binding site is located (Dever et al., 1987). The analysis of domain organization using SMART program (Schultz et al., 1998) showed that the consensus sequences GHVDHGKT (18-25), DCPG (80-83), and NKCD (135-138) involved in three GDP binding domains of $E$. coli EF-Tu (Kjeldgaard and Nybrog, 1992) are conserved in bEF1 $\alpha$ at amino acid residues 14-22, 91-94, and 153-156, respectively (Fig. 2).

\section{Characterization of bEF1 $\alpha$ mRNA expression}

To confirm whether bEF1 $\alpha$ mRNA was ubiquitously expressed or not in B. mori, RT-PCR was performed. There were a little difference in expression level of the $b$ bEF $1 \alpha$ mRNA according to each tissue and developmen- 
Fig. 2. The genomic nucleotide and deduced aa sequences of B. mori elongation factor $1 \alpha$ (bEF1 $\alpha$ ) gene (GenBank accession number: JQ638952). Exons are indicated by underlines. The transcription start site was defined as position +1 . The shaded areas indicate consensus sequence of GDP binding domains of $E$. coil. The predicted amino acid sequences (single-letter abbreviation) are shown below the nucleotide sequences. The asterisks indicate translation start and stop codon, and putative polyadenylation signal, respectively.

tal stage of 5th instar larvae, but the bEF1 $\alpha$ mRNA was ubiquitously expressed in all tissues and developmental stages tested (Fig. 1). As these results, we suppose that bEF $1 \alpha$ cDNA has potential of development as a valuable promoter for ubiquitous and powerful expression of a heterologous gene.

\section{Structural analysis of bEF1 $\alpha$ genomic DNA}

A positive phage clone was isolated from the screening of a $B$. mori genomic DNA library with bEF1 $\alpha$ cDNA probes labeled with $\left[\alpha-{ }_{-}^{32} \mathrm{P}\right]$ dATP. It was including the complete genomic sequence containing 906 bp of the 5'flanking region (GenBank accession number; JQ638952) 
A

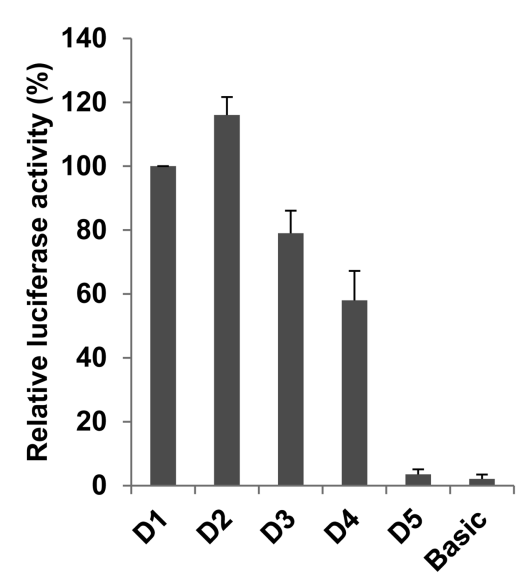

C

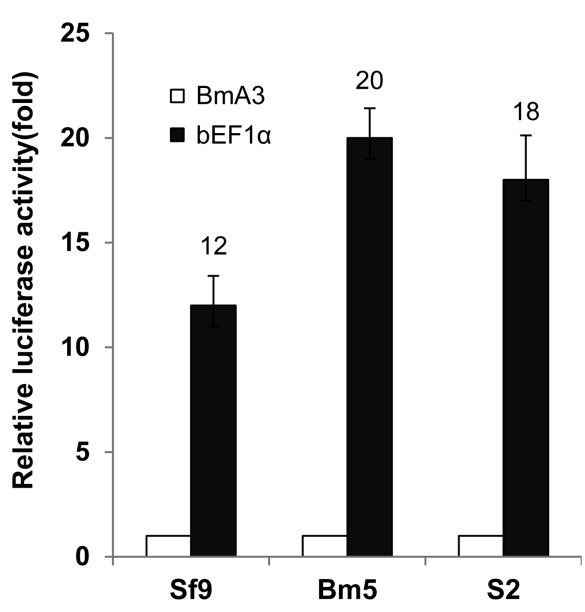

B

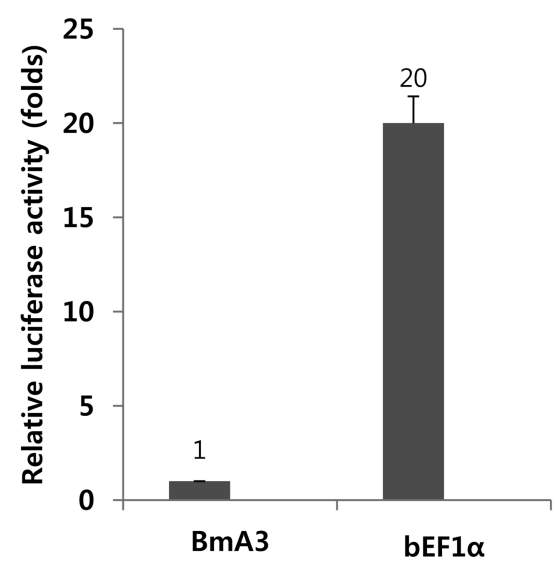

Fig. 3. Analysis of bEF $1 \alpha$ deletion constructs by dual luciferase reporter gene activity. (A) Various lengths of 5'-DNA sequences of bEF1 $\alpha$ were linked to a promoterless luciferase reporter plasmid, pGL3-Basic. These deletion constructs were used for the transient transfection into Bm5 culture cells. Luciferase activity by pGL3-bEF1 $\alpha-\mathrm{D} 1(-905 /+38)$ was defined as $100 \%$. (B) Comparison of luciferase activity with BmA3 promter. Luciferase activity by pGL3-BmA3 was defined as 1 fold. (C) Promoter activity of bEF1 $\alpha$ gene in insect lines, $\mathrm{Sf} 9, \mathrm{Bm} 5$ and $\mathrm{S} 2$ cells. Luciferase activity by pGL3-BmA3 was defined as 1 fold. The mean luciferase activity of deletion constructs was based on at least three independent experiments performed in triplicate, each normalized to an internal control. The bars represent standard deviation of each mean.

(Fig. 2). bEF1 $\alpha$ genomic DNA was composed of two exons of 38 and 1,633 bp, separated by two introns of 906 and $588 \mathrm{bp}$, respectively. The canonical GT/AG splice sites were present at the extreme ends of the intron (Fig. 2). The transcription start site was identified with 5'RACE PCR, because insect specific cap site, ATCAT(G) [19] and ATCATAC [14] was not found in bEF1 $\alpha$ gene. The putative transcription start site was located $49 \mathrm{bp}$ upstream from the ATG, the translation start codon and defined as position +1 (see Prediction for transcription start site in Materials and Methods) (Fig. 2). A putative TATA and CAAT box were not founded in bEF1 $\alpha$ gene.

\section{Activity of bEF1 $\alpha$ promoter}

To investigate the activity of bEF1 $\alpha$ promoter, a $944 \mathrm{bp}$ bEF1 $\alpha$ genomic DNA between -905 and +38 bp containing exon 1 was cloned into promoterless pGL3-basic vector (Promega, USA). A series of deletion fragments were made from this plasmid using some primers (Table 1), and cloned into $M l u$ I and Xho I sites of pGL3-Basic vector. The resulting deletion constructs, pGL3-bEF1 $\alpha-D 1$ (-905/ +38), pGL3-bEF1 $\alpha$-D2 (-702/+38), pGL3-bEF1 $\alpha$-D3 ($504 /+38)$, pGL3-bEF1 $\alpha-D 3(-244 /+38)$, and pGL3bEF1 $\alpha$-D5 $(-98 /+38)$ were co-transfected with control plasmid into Bm5 cells. The promoter activity of each 
construct was evaluated by measuring luciferase activity in comparison with a co-transfected, pRL-dHsp70-Rluc control plasmid. When the luciferase activity of the longest construct, pGL3-bEF1 $\alpha$-D1 was designated as $100 \%$, the relative promoter activity (\%) of other deletion constructs to pGL3-bEF1 $\alpha-D 1$ was shown in Fig. 3A. Among these 5 deletion constructs, the second construct, pGL3-bEF1 $\alpha$-D2 had the highest effect on transcriptional activity, with approximately $116 \%$ activity compared to pGL3-bEF1 $\alpha$-D1. The transcriptional activities of other deletion constructs from pGL3-bEF1 $\alpha-\mathrm{D} 3$ to pGL3bEF1 $\alpha$-D5 were gradually decreased in a size-dependent manner. The pGL3-bEF1 $\alpha$-D5 had no promoter activity with similarity to pGL3-Basic vector Fig. 3A. Accordingly, we selected the $\mathrm{D} 2$ region between -702 and +38 as the most suitable promoter region for expression of heterologous genes.

The promoter activity of pGL3-bEF1 $\alpha$-D2 was evaluated in comparison with $\mathrm{BmA} 3$ promoter which have used for ubiquitous expression of foreign genes in transgenic silkworm. The promoter activity of pGL3-bEF1 $\alpha-\mathrm{D} 2$ was 20.1 fold higher than that of BmA3 promoter (Fig. 3B).

To confirm whether the bEF1 $\alpha$ promoter also is normally regulated at other insect cell lines, the pGL3bEF1 $\alpha-\mathrm{D} 2$ and pGL3-BmA3 plasmids were transfected into $\mathrm{Bm} 5, \mathrm{Sf} 9$ and $\mathrm{S} 2$ insect cells, and then their promoter activity was measured, respectively. The bEF $1 \alpha$ promoter normally worked in $\mathrm{Bm} 5, \mathrm{Sf} 9$ and $\mathrm{S} 2$ cells, and had higher transcriptional activity than $\mathrm{BmA} 3$ in all insect cells tested (Fig. 3C). From the above results, we suggest that $b E F 1 \alpha$ promoter is more powerful and effective for construction of the transgenic silkworm or cell expression system due to higher transcription activity than that of BmA3. Furthermore, it can be used for heterogonous protein expression in various host insect as a universal promoter.

\section{Acknowlegements}

This research was supported by Bio-industry Technology Development Program (grant no. 311059-4), Ministry for Food, Agriculture, Forestry and Fisheries, Republic of Korea.

\section{References}

Ailor E, Betenbaugh MJ (1999) Modifying secretion and posttranslational processing in insect cells. Curr Opin Biotechnol 10, 142-145.

Dever TE, Glynias MJ, Merrick WC (1987) GTP-binding domain: Three consensus sequence elements with distinct spacing. Proc Natl Acad Sci U S A. 84, 1814-1818.

Goo TW, Yun EY, Hwang JS, Kang SW, Park S, You KH, Kwon OY (2002) Molecular characterization of a Bombyx mori protein disulfide isomerase (bPDI). Cell Stress Chaperones. 7, 118-125.

Goo TW, Kim SW, Kim SR, Park SW, Kang SW, Lee KG, Kwon OY, Yun EY (2010) Utilization of the Bombyx mori Hypothetical Protein 32 Promoter for Efficient Transgene Expression. Int J Indust Entomol 20, 107-114.

Goo TW, Kim SW, Kim YB, Kim SR, Park SW, Kang SW, Kwon OY, Yun EY (2011) A Powerful Ubiquitous Activity of Bombyx mori Heat Shock Protein 70 Promoter. Genes \& Genomics 33, 635-643

Handler AM, McCombs SD, Fraser MJ Jr, Saul SH (1998) The Lepidopteran transposon vector, piggyBac, mediates germline transformation in the Mediterranean fruit fly. Proc Natl Acad Sci USA 9513, 7520-7525.

Hershey JW (1991) Translational control in mammalian cells. Annu Rev Biochem 60, 717-755.

Horn C, Schmid BGM, Pogoda FS, Wimmer EA (2002) Fluorescent transformation markers for insect transgenesis. Insect Biochem Mol Biol 32, 1221-1235.

Kato T, Kajikawa M, Maenaka K, Park EY (2010) Silkworm expression system as a platform technology in life science. Appl Microbiol Biotechnol 85, 459-470.

Kjeldgaard M, Nyborg J (1992) Refined structure of elongation factor ef-tu from escherichia coli. J Mol Biol 223, 721742.

Mangé A, Julien E, Prudhomme JC, Couble P (1997) A strong inhibitory element down-regulates SRE-stimulated transcription of the A3 cytoplasmic actin gene of Bombyx mori. J Mol Biol 265, 266-274.

Royer C, Jalabert A, Da Rocha M. Grenier AM, Mauchamp B, Couble P, Chavancy G (2005) Biosynthesis and cocoonexport of a recombinant globular protein in transgenic silkworms. Transgenic Res 14, 463-472.

Schultz J, Milpetz F, Bork P, Ponting CP (1998) Smart, a simple modular architecture research tool: Identification of signaling domains. Proc Natl Acad Sci USA 95, 5857-5864.

Summers MD, Smith GE (1987) A methods for baculovirus vector and insect cell culture procedures, Texas Agricultural Experiment Station, Bulletin No. 1555.

Sun SC, Lindstrom I, Boman HG, Faye I, Schmidt O (1990) Hemolin: an insect-immune protein belonging to the immunoglobulin superfamily. Science 250, 1729-1732.

Tamura T, Thibert C, Royer C, Kanda T, Abraham E, Kamba M, Komoto N, Thomas JL, Mauchamp B, Chavancy G, Shirk P, Fraser M, Prudhomme JC, Couble P (2000) Germline transformation of the silkworm Bombyx mori L. using a piggyBac transposon-derived vector. Nat Biotechnol 18, 8184.

Thomas JL, Da Rocha M, Besse A, Mauchamp B, Chavancy G (2002) 3xP3-EGFP marker facilitates screening for transgenic silkworm Bombyx mori L. from the embryonic stage 
onwards. Insect Biochem. Mol Biol 32, 247-253.

Tomita M, Munetsuna H, Sato T, Adachi T, Hino R (2003) Transgenic silkworms produce recombinant human type III procollagen in cocoons. Na. Biotechno. 21, 52-56.

Tomita M, Hino R, Ogawa S, Iizuka M, Adachi T, Shimizu K, Sotoshiro H, Yoshizato K (2007) A germline transgenic silkworm that secretes recombinant proteins in the sericin layer of cocoon. Transgenic Res 16, 449-465.

Tryselius Y, Samakovlis C, Kimbrell DA, Hultmark D (1992) $\mathrm{CecC}$, a cecropin gene expressed during metamorphosis in Drosophila pupae. Eur J Biochem 204, 395-399.

Uchino K, Imamura M, Shimizu K, Kanda T, Tamura T (2007) Germ line transformation of the silkworm, Bombyx mori, using the transposable element Minos. Mol. Genet. Genomics 277, 213-220.

Uhlírová M, Asahina M, Riddiford LM, Jindra M (2002) Heat- inducible transgenic expression in the silkmoth Bombyx mori. Dev Genes Evol 212, 145-151.

Wurm FM (2003) Human therapeutic proteins from silkworms. Nat. Biotechnol. 21, 34-35.

Xue R, Chen H, Cui L, Cao G, Zhou W, Zheng X, aGong C (2012) Expression of hgm-csf in silk glands of transgenic silkworms using gene targeting vector. Transgenic Res. 21, 101-111.

Yun EY, Goo TW, Kim SW, Choi KH, Hwang JS, Kang SW, Kwon, OY (2005) Galatosylation and sialylation of mammalian glycoproteins produced by baculovirus-madiated gene expression in insect cells. Biotechnol Lett 7, 035-7039.

Zhao A, Zhao T, Zhang Y, Xia Q, Lu C, Zhou Z, Xiang Z, Nakagaki M (2010) New and highly efficient expression systems for expressing selectively foreign protein in the silk glands of transgenic silkworm. Transgenic Res. 19, 29-44. 